\title{
Anti-Corrosion Activity of Ephedrine on Mild Steel in Acidic Medium
}

\author{
Shaimaa Basim Khalaf Al-Baghdadi \\ Applied Science Department, University of Technology, Baghdad-Iraq. \\ E-mail: shaimaalbaghdadi@yahoo.com
}

\begin{abstract}
Anti-corrosion activity of ephedrine "2-(methylamino)-1-phenylpropan-1-ol" was investigated by Gravimetric strategy and completed with mellow steel samples in $1 \mathrm{M} \mathrm{HCl}$ as corrosive solution at different temperatures. The target compound displayed greatest efficiency of $84 \%$ at $500 \mathrm{mg} / \mathrm{L}$. The outcomes of weight loss method revealed that the target compound represents a one of the best inhibitors for mild steel and these efficiencies expanded with expansion in the concentrations of the ephedrine. SEM (Scanning Electron Microscopy) was utilized examine the steel surface at the most noteworthy erosion hindrance investigated concentration. [DOI: 10.22401/JNUS.20.1.09]
\end{abstract}

Keywords: corrosion, methylamino, phenylpropan, weight loss.

\section{Introduction}

Mild steel is having enormous applications in industries because of accessibility and low cost. Also it used in fabrication of different reactions like oil pipelines. [1]. Enormous published studies on corrosion inhibitors reveals that natural and synthesis organic compounds that applied as inhibitors for mild steel could adsorbed on the steel surface via the atoms nitrogen, oxygen, sulfur and phosphorus that called heteroatoms and/or double bond, aromatic ring and decreasing the corrosion rate [2-9].

In many industries the corrosion of mild steel were problems that incessantly attracted researchers in. Industries that using aggressive solutions like acids and bases in cleaning or etching or pickling causes corrosive of the mild steel surface. many methods and techniques like cathodic protection and anodic protection or coating or using organic or natural compounds as corrosion inhibitors have been utilized to diminish the corrosion of mild steel [10-13]. In the present study, ephedrine "2-(methylamino)-1-phenylpropan1-ol" was examine as green inhibitor of MS (Mild Steel) corrosion in $1 \mathrm{M}$ of $\mathrm{HCl}$ using weight-loss strategy in addition to SEM.

\section{Experimental}

All chemicals that used in this study were brushed from Sigma-Aldridge and were used without further purification.

\section{Weight loss method}

MS samples had been cut to shape different examples, with measurements $1.0 \mathrm{~cm} \times 2.0 \mathrm{~cm} \times 0.05 \mathrm{~cm}$. Specimens have been washed 2 times by distilled water then by acetone. Solutions of $\mathrm{HCl} 1.0 \mathrm{M}$ were utilized to be blank. Ephedrine as inhibitor with various concentrations 0.0, 100, 200, 300, 400 and $500 \mathrm{mg} / \mathrm{L}$., had been added to corrosive solutions. MS were submerged in $250 \mathrm{ml}$ of various measures of ephedrine for $6.0 \mathrm{hrs}$., of immersion every sample in range of temperatures $30,40,50$ and $60^{\circ} \mathrm{C}$. After that the samples were taken out an sodium hydroxide was utilized as washing a solution with zinc-dust then allowed to dry and weight accurate. \%IE with corrosion rate have been figured by utilizing the below equations(1-4):

$$
\% I E=\left(\frac{W_{2}-W_{1}}{W_{2}}\right) \times 100
$$

$\mathrm{W}_{1}$ with $\mathrm{W}_{2}$ represent the values of weight reduction without/with of ephedrine in corrosive solution. \%IE is the inhibition efficiency.

$$
C R=\frac{w}{A t}
$$

$\mathrm{w}$; weight loss. A; area of specimen. $\mathrm{t}$; immersion time. $\mathrm{CR}$; corrosion rate

$\theta=\left(\frac{C R_{2}-C R_{1}}{C R_{2}}\right) \times 100$

$\theta$ is the surface coverage

$$
\% I E=\theta \times 100
$$




\section{Morphology}

Scanning electron microscope (SEM) had been done and was coordinated at the University Kebangsaan Malaysia. Electron Microscopy Unit. Specimens were submerged in corrosive solution in presence/absence of $500 \mathrm{mg} / \mathrm{L}$., of ephedrine.

\section{Results and Discussion}

Natural inhibitors demonstrate cathodic/ anodic and plays through a procedure of adsorption and forming of coated films. Ephedrine, show a solid affinity for MS surface that could prompt to forming coordination compounds having benefit of helpful restraint action and decreasing dangerous [14]. Ephedrine gives defensive hydrophobic film barrier that adsorb molecule on surface of MS.

\section{Weight loss technique}

Ephedrine at different concentrations had been shown by Fig.(1), in $1.0 \mathrm{M}$ corrosive solution without/ with of ephedrine. CR in corrosive solution without ephedrine had been increased sharply see in Fig.(2). Expanding of ephedrine concentration lead to decreasing CR sharply at various Temperatures.

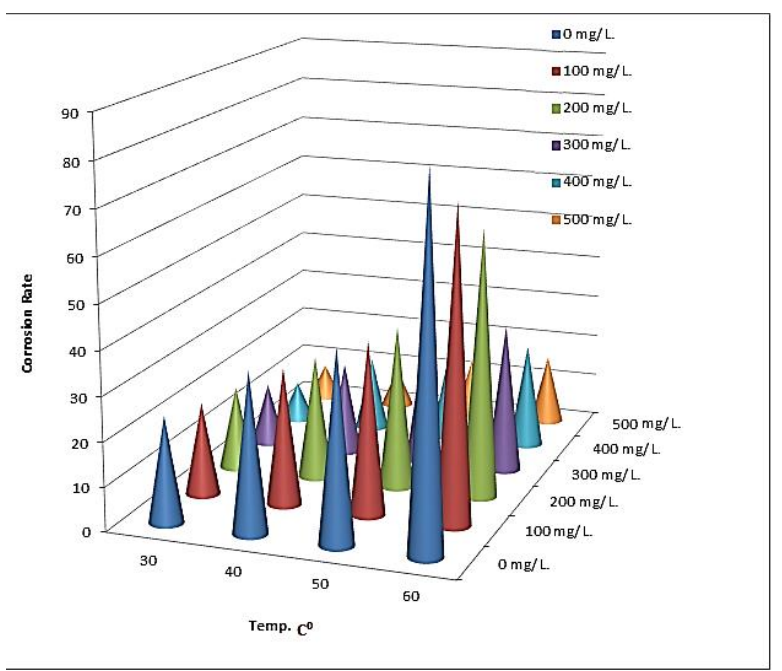

Fig.(1): The CR for the MS with various concentrations of ephedrine at different temperatures.

IE\% increase positively with ephedrine concentrations. Fig.(2), represent the inhibition efficiency diminish with raising of temperature and increase the restraint productivity with expansion the concentration. The positively increasing of efficiency with ephedrine was because of the expanding in adsorption on MS surface of the ephedrine with higherconcentration or expanding of complexity between ephedrine molecules and MS by means of coordination-bonds. Experimentaltemperature $60 \mathrm{C}^{0}$ the efficiency was the lowest that could might be related to decreasing of stability of molecules of inhibitor due to raising of temperatures.

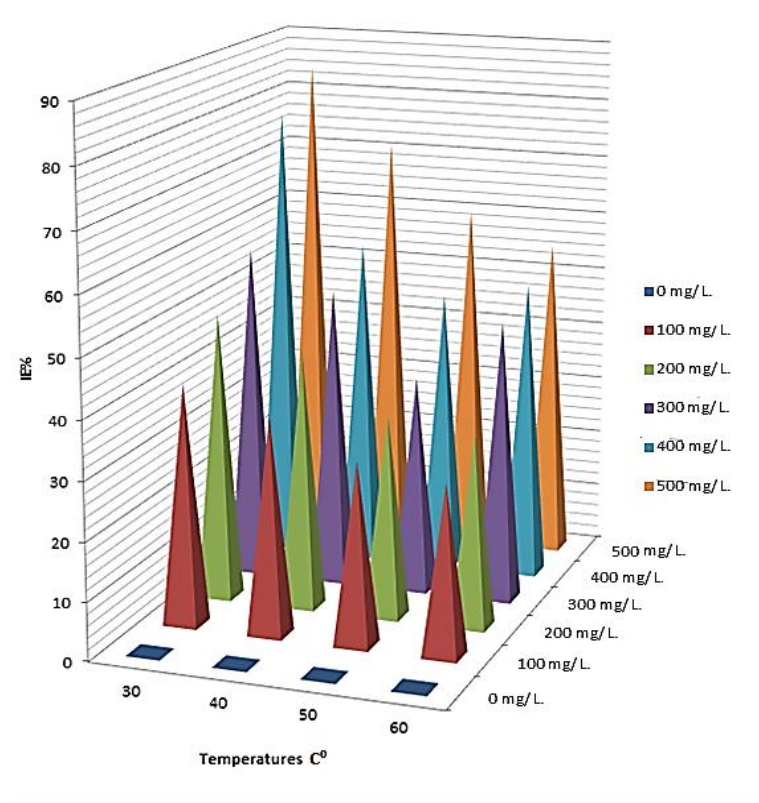

Fig.(2): Inhibition efficiency for $M S$ corrosion at various temperatures for ephedrine with various concentrations.

\section{Adsorption Isotherm}

Langmuir isotherm was appeared by adsorption isotherm [15] of CR information. The adsorption isotherm balance consistent was good pointer for mechanisms of restraint, so if the estimation of adsorption isotherm equilibrium steady has been high in other word strong adsorbed on MS.

$$
\theta=\frac{K c}{K c+1}
$$

Where $\mathrm{K}$, equilibrium constant of adsorption isotherm. C, ephedrine concentration.

Rearrangement equation 5

$\theta(K c+1)=K c$

Dividing by c $\theta$, yield

$$
K+\frac{1}{c}=\frac{K}{\theta}
$$


Multiply by $\mathrm{C} / \mathrm{K}$

$$
\mathrm{c}+\frac{1}{\mathrm{~K}}=\frac{\mathrm{c}}{\theta}
$$

To demonstrate that adsorptions system were by Langmuir/adsorption isotherms for various temps., in corrosive solutions we plot Fig.(3), that showed c/ $\theta$ vs. c. Straight/line demonstrating ephedrine molecules were adsorbed by Langmuir/adsorption isotherms.

Where $1 / \mathrm{K}$, intercept.

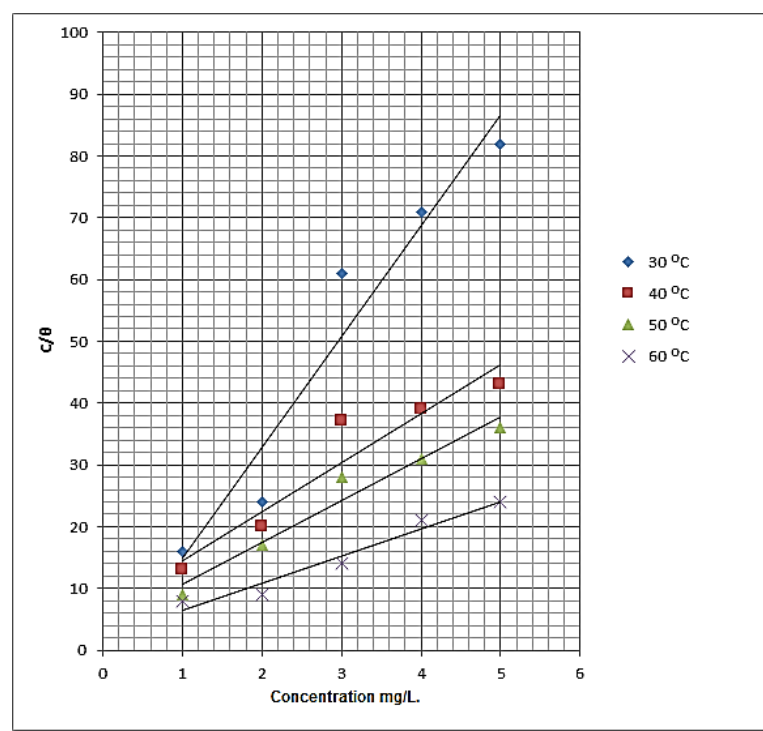

Fig.(3): Langmuir/plot for the ephedrine.

\section{Surface Morphology}

Fig.(4) demonstrate MS surface that immersed in acidic solution without/with the ephedrine. Making a rougher surface was done by utilizing $1 \mathrm{M} \mathrm{HCl}$. The differences were shown from Fig.(4) in corrosive solutions with smoother surface because of ephedrine. The smooth surface in Fig.(4b) was due to the ability of ephedrine as inhibitor to reduce the corrosion and represent adsorb films on morphology for the MS (mild steel) surface that not seen in Fig.(4). Mechanism could clarify based on one of the accompanying reasons. Ephedrine is adsorbed (chemisorption) on MS surface and structures a defensive film with inhibitor impact or by mix between inhibitor particles and metallic surface. The chemical reaction between ephedrine and surface of MS as a way that the metal with d-orbital so, in this way bond will be formed through forming of coordination bond between the ephedrine and MS on the basis that ephedrine represents a ligand contain hetro-atoms and electronic pairs and additionally aromatic ring. Electrons could combine and forming the coordinate complex with coordinate bonds by transferring electrons from the Ephedrine to unoccupied/orbitals (d) for MS the metal by.

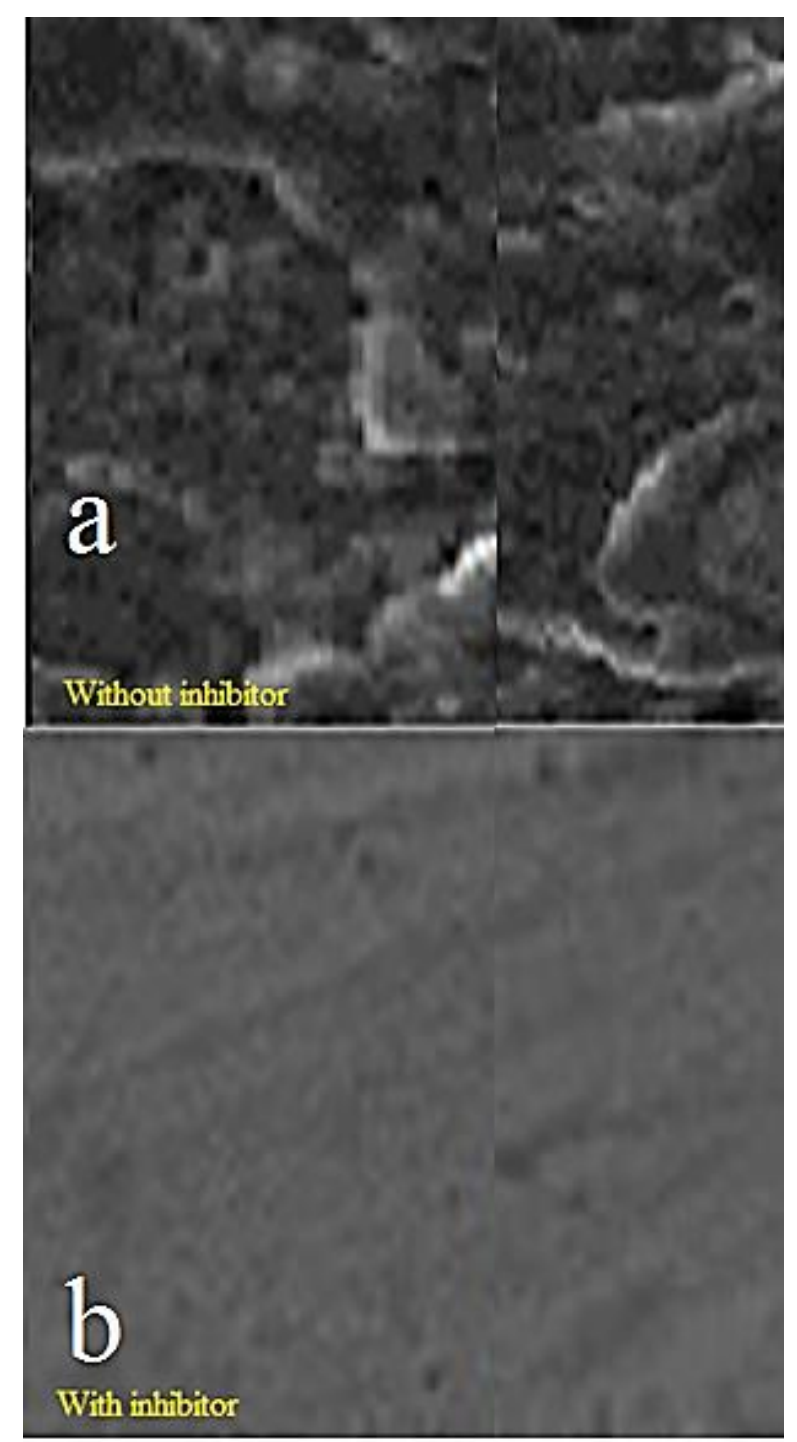

Fig.(4): SEM for MS in presence/absence ephedrine.

\section{Conclusions}

The corrosion inhibitor ephedrine was found to be a good inhibitor of mild steel in acidic solutions. Efficiencies were increasing with increasing of concentration of ephedrine and decreasing with raising of temperature, and had inhibition efficiency of $84 \%$.

\section{References}

[1] Ramesha S., Rajeswari S., Maruthamuthu S. "Effect of inhibitors and biocide on corrosion control of mild steel in natural 
aqueous environment", Materials Letters 57, 4547 - 4554, 2003

[2] Hui-Long W., Hong-Bo F., and Jia-Shen Z., "Corrosion inhibition of mild steel in hydrochloric acid solution by a mercaptotriazole compound", Materials Chemistry and Physics 77, 655-661, 2003.

[3] Shyamala M., Arulanantham A., "Corrosion Inhibition of Centella asitica (Vallarai) on Mild Steel in Hydrocloric Acid", Asian Journal of Chemistry, 21, 6102-6110, 2009.

[4] Umoru L.E, A.Fawehinmi and A.Y. Fasasi, "Investigation of the Inhibitive Influence of Theobroma Cacao and Cola Acuminata Leaves Extracts on the Corrosion of a Mild Steel in Sea Water", Journal of Applied Sciences Research, 2, 299-304, 2006.

[5] Lebrini M., Robert F., Ross C., Alkoloids "Extract from Palicourea guianensis Plant as Corrosion of C38 Steel in $1 \mathrm{M}$ Hydrochloric Acid Medium", Int. J. Electrochem. Sci, 6, 847-859, 2011.

[6] Ostovari. A, Hoseinieh, S.M, Peikari, M, Shadizadeh, S.R, Hashemi, S.J, "Corrosion Inhibitor of Mild Steel in $1 \mathrm{M} \mathrm{HCl}$ solution by Henna Extract: A Comparative Study of Inhibition by Henna and Its Contituents (Lawsone, Gallic acid, Glucose and Tannic Acid)", Corrosion Science, 8, 342-350, 2009.

[7] Aprael S. Yaro, Anees A. Khadom, Rafal $\mathrm{K}$. Wael, "Apricot juice as green corrosion inhibitor of mild steel in phosphoric acid," Alexandria Engineering Journal, 52, 129135, 2013.

[8] Hmamou, D.B. Salghi, R. Zarrouk, A. Messali, M. Zarrok, H. Errami, M. Hammouti, B. Bazzi, L. Chakir, A. "Inhibition of steel corrosion in hydrochloric acid solution by chamomile extract", Der Pharma Chem. 4, 1496-1505, 2012.

[9] Yousif E., Win F., Al-Hamadani H., AlAmiery A., Kadhum A., Mohamad A., "Furosemide as an Environmental-Friendly Inhibitor of Corrosion of Zinc metal in Acid Medium: Experimental and Theoretical studies", Int. J. Electrochem. Sci 10, 1708$1715,2014$.
[10] Al-Amiery. A., Abdul, A., Abu M., Sutiana, J., "A novel hydrazinecarbothioamide as a potential corrosion inhibitor for mild steel in $\mathrm{HCl}$ ", Materials, 6, 1420-1431, 2013.

[11] Junaedi, S., Al-Amiery A., Kadihum A., Mohamad A., "Inhibition effects of a synthesized novel 4-Aminoantipyrine derivative on the corrosion of mild steel in hydrochloric acid solution together with quantum chemical studies" Int. J. Mol. Sci, 14, 11915-11928, 2013.

[12] Kadhum, A. Mohamad, A. B. Hammed, L. Al-Amiery A. A., San, N. H., Musa A. Y. Inhibition of Mild Steel Corrosion in Hydrochloric Acid Solution by New Coumarin. Materials, 7, 4335- 4348, 2014.

[13] Al-Amiery, A. A. Novel Corrosion Inhibitor for Mild Steel in $\mathrm{HCl}$. Materials, 7, 662-672, 2014.

[14] Rubaye A. Y., Abdulwahid A., AlAmiery A., Kadhum A., and Mohamad A., Cheery Sticks Plant Extract as a Green Corrosion Inhibitor Complemented with LC-EIS/ MS Spectroscopy. Int. J. Electrochem. Sci., 10 (9), 8200 - 8209, 2015.

[15] Al-Amiery, Y. Al-Majedy, A. Kadhum, A. Mohamad, A. New Coumarin Derivative as an Eco-Friendly Inhibitor of Corrosion of Mild Steel in Acid Medium, Molecules, 20, 366-383, 2015. 